\title{
Tumor location determines midkine level and its association with the disease progression in colorectal cancer patients: a pilot study
}

\author{
Malgorzata Krzystek-Korpacka • Dorota Diakowska • \\ Krzysztof Grabowski • Andrzej Gamian
}

Accepted: 13 April 2012 / Published online: 6 May 2012

(C) The Author(s) 2012. This article is published with open access at Springerlink.com

\begin{abstract}
Purpose The purpose of this study was to evaluate midkine, multipotential cytokine, and growth factor in colorectal cancer (CRC) stratified by tumor location.

Methods Midkine was assessed immunoenzymatically in paired cancerous and noncancerous tissues from 53 CRCs and referred to CRC stage, tumor location, and size, and circulating cytokine levels.

Results Midkine was higher in cancerous versus noncancerous tissue in $98 \%$ cases $(424.2$ vs. $31.1 \mathrm{pg} / \mathrm{mg}, p<0.0001)$. Mean fold increase was 30.1 ; in $72.5 \%$, the relative increase was over fivefold. Midkine upregulation was more pronounced in colon than in rectum (fold increase: 36.6 vs. $12.7, p=0.005)$ due to higher midkine level in noncancerous rectal than colonic tissue (45.5 vs. $26.2 \mathrm{pg} / \mathrm{mg}, p=0.074$ ). Tumor location affected midkine association with CRC stage. Midkine fold change was higher in advanced stages of rectal cancers (16.8 vs. 5.3, respectively in III/IV vs. I/II, $p=0.013)$, while it tended to be lower in colonic ones ( 25.3 vs. $47.8, p=0.134$ ). In addition, fold change in midkine level was higher in rectal $\mathrm{N} 1$ than $\mathrm{N} 0$ cancers (17.3 vs. $16.5, p=$
\end{abstract}

M. Krzystek-Korpacka $(\bowtie) \cdot$ A. Gamian

Department of Medical Biochemistry,

Wroclaw Medical University,

ul. Chalubinskiego 10,

50-368 Wroclaw, Poland

e-mail: malgorzata.krzystek-korpacka@am.wroc.pl

D. Diakowska $\cdot$ K. Grabowski

Department of Gastrointestinal and General Surgery,

Wroclaw Medical University,

Wroclaw, Poland

A. Gamian

Wroclaw Research Center EIT+,

Wroclaw, Poland
0.032), while it tended to be lower in colonic cancers ( 23.6 vs. 50.1, $p=0.085)$. Midkine negatively correlated with tumor size $(r=0.40, p=0.017)$, while it tended to positively correlate with its serum levels $(r=0.45, p=0.081)$.

Conclusions Midkine is differently expressed in tumors arising from colonic and rectal mucosa, where it may play diverse roles in carcinogenesis. High midkine expression in noncancerous rectal mucosa might contribute to, a characteristic for rectal cancers, higher incidence of local recurrence. Divergent expression of midkine and its association pattern ought to be taken into account while designing midkine-directed therapies for CRC.

Keywords Midkine $\cdot$ Colorectal cancer $\cdot$ Lymph node metastasis $\cdot$ Cytokine $\cdot$ Growth factor

\section{Introduction}

Colorectal cancer (CRC) is a malignant tumor arising from the inner wall of large intestine. Worldwide, it is the third cause of cancer deaths in women and fourth in men and the second and the third most frequently diagnosed cancer in women and men, respectively. Unlike in USA and Western Europe, CRC incidence in Eastern European countries is increasing probably as a result of "westernization" of a lifestyle [1].

Individual segments of the colon differ with respect to their embryological origin, innervation, blood supply, lymphatic drainage, histology, physiological function, and content. It seems logical that tumors that arise from proximal and distal colon or, taking into account differences in histology and metabolism, from proximal and distal colon and rectum ought to be treated as distinct entities [2]. Indeed, it has been increasingly recognized that tumor location determines risk and 
protective factors, mechanisms promoting the disease progression, recurrence pattern, prognosis, and effectiveness of various treatment strategies [2-5]. Unveiling differences in cancer-related molecular patterns with respect to tumor location might aid future targeted therapies for CRC.

Midkine is a multifunctional cytokine predominantly expressed during embryogenesis, while in adult organisms, its expression is restricted to several organs, intestine, among others. However, midkine expression is resumed during inflammation, tissue repair, and carcinogenesis. Midkine displays a number of activities that might be relevant for cancer development, e.g., it has been demonstrated to act as a mitogen, an antiapoptotic, and angiogenic factor, a chemoattractant and haptotactic factor, an immunomodulator, and an inductor of synthesis of several cytokines and growth factors, such as IL-8, TGF- $\beta$, MIP-2, and MCP-1 $[6,7]$. Data on midkine in $\mathrm{CRC}$ are scanty and focused on its involvement in colonic neoplastic transformation [8-12], while data on cytokine levels in CRC are missing. Possible midkine contribution to divergence in molecular patterns involved in promoting tumor progression between CRC with different location has not been evaluated before as well. Hence, the aim of our study was to examine protein level of midkine in tumor versus normal colorectal tissue and relate it to the disease progression, tumor location, and circulating levels of cytokine.

\section{Materials and methods}

\section{Patients}

A group of 53 patients admitted between 2005 and 2008 to the Department of Gastrointestinal and General Surgery of Wroclaw Medical University for curative resection of histopathologically confirmed adenocarcinoma of the colon has been enrolled in current study. Open colectomy (right hemicolectomy $n=10$; transverse colectomy $n=7$; left hemicolectomy $n=3$; sigmoidectomy $n=19$; rectectomy $n=14$ ) was performed in 27 men and 26 women, mean age of 67.8 (range, 36-87). Resected tumors were staged pathologically according to the guidelines of the UICC TNM [13] system. There were 11 cases of T2, 10 cases of T3, and 30 cases of T4, and in two cases, $\mathrm{T}$ was not established (Tx); there were 25 cases of $\mathrm{N} 0$, 22 cases of $\mathrm{N} 1$, three of $\mathrm{N} 2$, and three of $\mathrm{Nx}$; no distant metastases were found in 47 cases, hepatic metastases in five cases, and in one, $\mathrm{M}$ status was uncertain. When grouped, we had eight patients with stage I, 17 with stage II, 20 with stage III, five with stage IV, and in three, the disease stage could not be assessed. The tumor was located in rectum in 14 cases, in colon in 36 cases (in ascending colon in seven, in transverse colon in seven, in descending colon in three, and in sigmoid colon in 19 cases), and in cecum in three cases. Two patients were excluded from the analysis following pathological examination owing to cancerous tissue present in resection margins.

The study protocol was approved by the Medical Ethics Committee of Wroclaw Medical University, Wroclaw, Poland, and the study was conducted in accordance with the Helsinki Declaration of 1975, as revised in 1983, and an informed consent has been obtained from all patients.

Analytical methods

A paired tissue samples, one representing tumor and the other noncancerous tissue from the same location (resection margin), have been collected postoperatively for each patient, snap frozen, and stored at $-45^{\circ} \mathrm{C}$ until examination. Frozen tissues $(\sim 0.37 \mathrm{~g})$ were placed in $10 \mathrm{mM}$ Tris- $\mathrm{HCl}$, pH 7.2 buffer $1: 2(w / v)$ and homogenized using a Potter homogenizer. Following centrifugation $(10 \mathrm{~min}, 1,850 \times \mathrm{g}$, $4^{\circ} \mathrm{C}$ ), supernatants were collected and used for subsequent midkine and protein evaluations. Additionally, serum samples (blood collected by venipuncture prior to surgery, clotted for $30 \mathrm{~min}$, and centrifuged $10 \mathrm{~min}, 900 \times \mathrm{g}$, RT) were available for a subset of 19 patients. Midkine concentrations in tissue homogenates and serum samples were measured with by an enzyme double-antibody indirect immunoassay using Human Midkine ELISA provided by Biovendor (Brno-Modřice, Czech Republic) according to manufacturer's instructions. An intra-assay coefficient of variation (CV) for this assay is $4.5 \%$ and inter-assay $\mathrm{CV}$ is $6.3 \%$, while the test sensitivity is $33 \mathrm{pg} / \mathrm{ml}$. Protein concentration in tissue homogenates was measured using Bradford method [14] with Bio-Rad Protein Assay (Bio-Rad Laboratories $\mathrm{GmbH}$, Munchen, Germany) with bovine serum albumin as the reference. Midkine concentration was expressed in picograms per milligram of total protein.

\section{Statistical analysis}

Data distribution was tested using the Kolmogorov-Smirnov normality test and homogeneity of variances using Levene's test. Data on midkine in cancerous tissue were normally distributed, while those in adjacent noncancerous tissue required logarithmic transformation; both are presented as means with $95 \%$ confidence interval. Differences in midkine level between cancerous and normal tissues were analyzed using $t$-test for paired samples. Differences in midkine level or fold change in groups stratified by the disease stage or tumor location were examined using $t$ test for independent samples (with Welch correction in case of unequal variances) and one-way ANOVA. Correlation analysis was conducted using Pearson correlation $(r)$ test, following removal of outlying observations detected using Tukey test (two outliers detected). All tests were two-sided and $p \leq 0.05$ were considered statistically significant. Entire statistical analysis was 
conducted using MedCalc ${ }^{\circledR}$ version 12.1.0.0 (Mariakerke, Belgium).

\section{Results}

Midkine in normal and tumor tissue

We compared midkine level in paired cancerous and normal tissue from patients with colon cancer and found mean midkine level to be significantly higher in cancerous tissue (Fig. 1). Except for one case, midkine was higher in tumor than in normal tissue, i.e., in $98 \%$ of the cases. In 37 out of $51(72.5 \%)$, the relative increase was over fivefold. Mean fold increase in midkine level was 30.1 (18.5-41.7).

\section{Midkine level and the disease progression}

Midkine level in cancerous tissue is negatively correlated with tumor size ( $r=-0.40, p=0.017$; regression line is presented in Fig. 2) and shows tendency to positively correlate with serum midkine concentrations $(r=0.45, p=0.081)$. There were no significant differences in midkine level between T2, T3, and T4 tumors $(p=0.754)$, in N0 vs. N1 disease $(p=0.732)$, in M0 vs. M1 disease $(p=0.485)$, and among stages I, II, III, and IV $(p=0.655)$.

\section{Midkine level and tumor location}

We observed higher midkine concentration in normal tissue derived from rectum $[45.5 \mathrm{pg} / \mathrm{mg}$ (26.5-78.3) than from colon (26.2 pg/mg (18.9-36.4), $p=0.074]$. When cancerous tissues from rectum and colon were compared, no differences in midkine concentration were found $(p=0.797)$. Consequently,

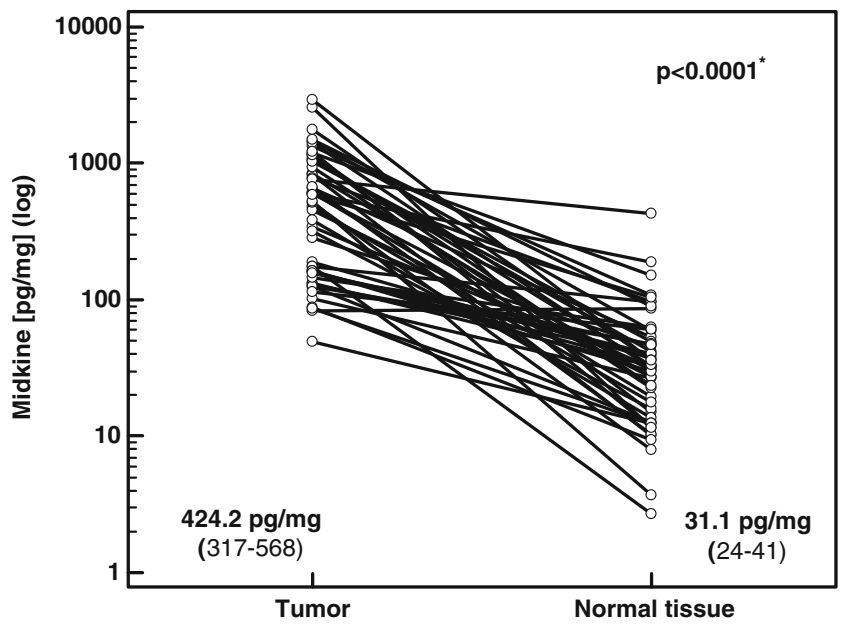

Fig. 1 Midkine level in cancerous and normal colon tissue in patients with colon cancer. Midkine level is expressed in picograms of midkine per miligram of total protein (means with $95 \%$ confidence intervals given in parentheses). Asterisk statistically significant

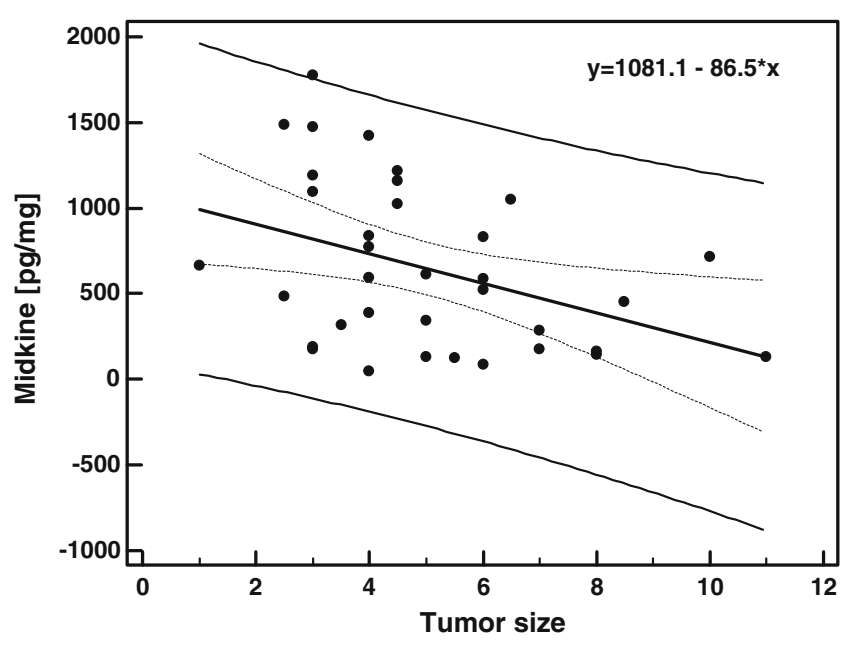

Fig. 2 Midkine relation to tumor size (regression line). Data on tumor size available for 36 patients; two outlying observations removed prior to analysis. $R^{2}=0.16, p=0.017$. Thick line regression curve, thinner lines $95 \%$ prediction interval curves, scattered lines $95 \%$ confidence interval

fold increase, i.e., ratio of midkine in cancerous to noncancerous tissue, was significantly higher in colon than in rectum (Fig. 3). Within colon, however, no difference between proximal and distal part could be observed, both when normal $(p=$ $0.862)$ or cancerous tissues $(p=0.839)$ were compared. The tendency towards higher fold change in midkine level in proximal than distal colonic tumors was not significant (45.6 times vs. $33, p=0.859$ ).

Midkine level and the disease progression in relation to tumor location

We re-examined the data by analyzing the fold changes in midkine level separately for colon and rectal cancers. The

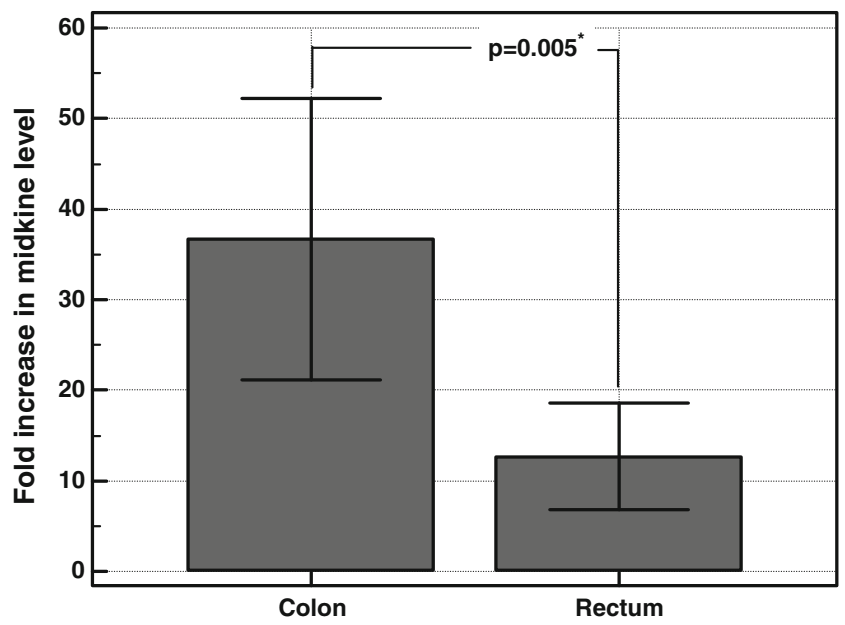

Fig. 3 Differences in fold increase in midkine level between cancerous and normal tissues in relation to tumor location. Whiskers indicate $95 \%$ confidence interval. Asterisk statistically significant 
fold change in midkine was significantly higher in advanced stages of rectal cancer, while the opposite trend was observed in colonic cancers (Fig. 4). In addition, fold change in midkine level was higher in rectal N1 than N0 cancers, while it tended to be lower in colonic cancers (Fig. 5). Similar tendency was found concerning $\mathrm{T}$ stage, where fold changes in midkine tended to be higher in T3/T4 than T2 rectal [15.6 (7-24) vs. $7(0.9-14), p=0.152]$ but not colonic tumors [37 (18-56) vs. 41 (5.6-77), $p=0.853]$. The number of patients with M1 disease was insufficient to allow for the statistical analysis of tumor-location stratified groups.

Difference in midkine association with the disease progression between rectal and colonic cancers was also observed, although less pronounced, when midkine levels in cancerous tissue and not fold changes were compared. Midkine level was significantly higher in advanced stages of rectal [636.5 pg/mg (314.5-1288) in III/IV vs. $190.8 \mathrm{pg} /$ $\mathrm{mg}(62-588)$ in I/II, $p=0.036]$ but not colonic cancers [respectively $409.5 \mathrm{pg} / \mathrm{mg}(227-740)$ vs. $514.5 \mathrm{pg} / \mathrm{ml}$ (308-858), $p=0.551]$. It tended to be higher in N1 vs. N0 disease in rectal [580 pg/mg (266-1265) vs. $264.2 \mathrm{pg} / \mathrm{mg}$ (80-871), $p=0.180]$ but not in colonic cancers $[375 \mathrm{pg} / \mathrm{mg}$ (212-664) vs. $553 \mathrm{pg} / \mathrm{mg}(330-928), p=0.301]$. Furthermore, midkine level tended to be lower in T3/T4 than T2 colonic tumors [respectively $399 \mathrm{pg} / \mathrm{mg}$ (265$601)$ vs. $833 \mathrm{pg} / \mathrm{mg}$ (475-1459), $p=0.146]$ but not rectal tumors [465 pg/mg (193-1117) vs. $336 \mathrm{pg} / \mathrm{mg}(98-1151)$, $p=0.606]$.

\section{Discussion}

To best of our knowledge, this is the first paper showing differences in midkine level as well as in its association

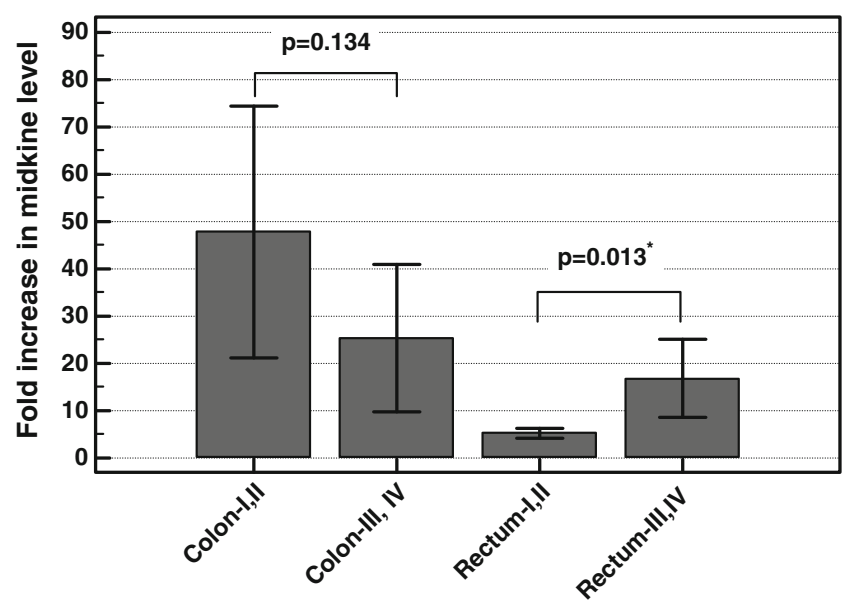

Fig. 4 Fold changes in midkine level between cancerous and normal tissue in relation to tumor location and the disease stage. Whiskers indicate $95 \%$ confidence interval. Asterisk statistically significant

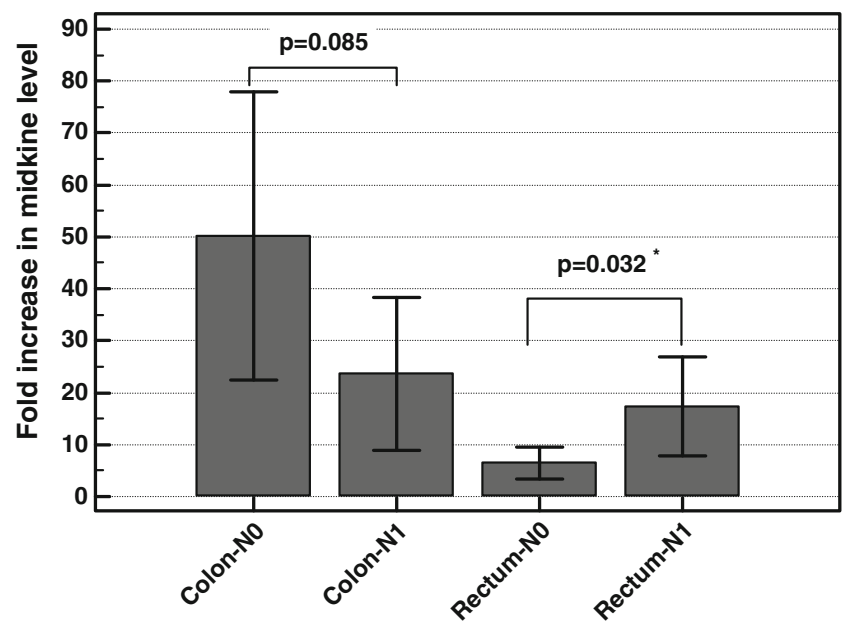

Fig. 5 Fold changes in midkine level between cancerous and normal tissue in relation to tumor location and lymph node involvement. Whiskers indicate $95 \%$ confidence interval. Asterisk statistically significant

pattern with the disease progression between cancers of colon and rectum.

Data on midkine in CRC are surprisingly scanty. Aridome et al. [8] were first to detect, semi-quantitatively, higher midkine expression in cancerous than noncancerous tissue from 12 out of 13 CRC patients (>92\%). Subsequently, Miyashiro et al. [9] confirmed midkine messenger RNA expression in cancerous tissues, either as a fullsize midkine or its truncated form, devoid of its N-terminal domain. Since the truncated midkine has been found in cancerous colorectal tissue and in metastatic lymph nodes, but not in adjacent noncancerous tissue, the cancer-specific nature of the truncated form has been suggested [10]. Corroborating with these original findings, we found higher midkine concentration in cancerous than noncancerous tissue in $98 \%$ of examined cases. Owing to the polyclonal character of the antibody used for midkine detection, we were likely to detect both full and truncated midkine forms.

Ye et al. [11] and Tokuyama et al. [12] demonstrated midkine expression to be higher in adenomas and adenocarcinomas than in normal mucosa, being significantly elevated already in low-grade [12] or moderate-grade dysplasia [11]. While Tokuyama et al. [12] observed midkine expression to increase in a stepwise manner, Ye et al. [11] found the highest midkine levels in adenomas. Midkine expression has corresponded with cell proliferative zone, that is, the labeling of Ki-67, a proliferative index [12]. Taken together, these results may imply that elevated midkine expression can contribute to early stages of colon carcinogenesis by facilitating tumor cell proliferation. The issue of midkine expression in relation to CRC stage has not been examined in earlier studies. Supporting the thesis on midkine involvement in early colon carcinogenesis and its possible 
downregulation in advanced cancers, we found a negative correlation between midkine concentration and tumor size but did not observed any other association with the disease advancement. However, further analysis revealed that midkine expression differed with respect to tumor location. Not only we found midkine to be expressed more profusely in noncancerous rectal than colonic tissue, but also tumor location determined midkine association with the disease stage. Midkine was upregulated in advanced cancers in rectum, being significantly associated with overall disease stage and lymph node involvement, but downregulated in advanced colonic cancers. Previously, Song et al. [15] observed serum midkine to be more frequently elevated in lymph node positive than negative colon cancers. The association of circulating midkine with lymph node metastasis has also been demonstrated in esophageal squamous cell carcinoma [16] and in endometrial cancers [17]. Our results, if confirmed in a larger cohort, might suggest divergent role of midkine in colonic and rectal carcinogenesis.

A body of evidence has recently been gathered documenting variability in molecular patterns of colorectal cancers located in various parts of large intestine. Proximal colonic tumors have been observed to overexpress Ki-67 and p53 [4], Bcl-2 (solely in female) [4], keratins, and carbonic anhydrases [18] while in distal colonic tumors COX2 and teratocarcinoma growth factor [18]. Tumors of proximal colon are also more likely to be poorly differentiated $[4,5,19]$ and associated with worse overall but not cancer-specific survival [5]. In turn, Derwinger et al. [5] reported differences within distal colonic cancers with cancers of sigmoid colon having more favorable prognosis expressed in terms of overall survival and cancerspecific survival, resulting from better stage and grade, as compared to descending colon. Correspondingly, fold change in midkine in descending colon tumors was higher than in sigmoid colon tumors, but as we examined only three cases of the former, the difference did not reached statistical significance (45 times vs. 31, data not shown). Minoo et al. [19] examined differences in expression patterns of 50 markers associated either with major signaling pathways involved in tumor progression or with immune response, of which several were more frequently overexpressed in rectal than other distal colon cancers (CD44v6, E-cadherin, CD68, CD163, and Foxp3), while others were found to be differently expressed in rectal vs. proximal cancers (TOPK, APAF-1, p21, Foxp3, TIA-1, CDX2, and $\beta$-catenin). In turn, Kalady et al. [20] found a higher incidence of microsatellite instability, methylator phenotype, and mutations in the oncogene $B R A F$ in colonic than rectal cancers. Microsatellite stable tumors had almost four times higher risk of disease recurrence [20], what may contribute to higher incidence of local disease recurrence and account for generally worse prognosis characteristic for rectal cancers. In this respect, higher baseline midkine expression in rectal mucosa together with a direct midkine association with disease progression might contribute to this feature of rectal cancers. It is worth mentioning that midkine has recently been suggested as the recurrence marker for desmoid tumors, monoclonal neoplasms that may occur as a part of familial adenomatosis polyposis [21].

Midkine overexpression is common among solid tumors, as it may facilitate tumor cell proliferation, survival, and migration, and enables invasion. As such, midkine might become a promising target for future directed therapies. A number of strategies to inhibit midkine expression/signaling are under investigation [22], and successful suppression of hepatocellular carcinoma growth by midkine-antisense oligonucleotide-loaded nanoparticles has already been reported [23]. However, regional divergence in midkine expression and, possibly, the role it might play in the disease progression ought to be taken into account while designing midkine-directed therapies for CRC.

Taking into account regional differences in midkine level, relative changes expressed in terms of fold changes rather than absolute cytokine levels should be evaluated in future studies. However, it would be of interest to compare midkine level in colonic and rectal tissues from noncancer patients to address the issue whether higher midkine level in noncancerous rectal than in colonic mucosa observed in $\mathrm{CRC}$ is disease related.

Acknowledgements Dr Malgorzata Krzystek-Korpacka received a research fellowship within "Development program of Wroclaw Medical University" funded from European Social Fund, Human Capital, national Cohesion Strategy" (contract no. UDA-POKL.04.01.01-00010/08-00)" and the research was in part supported by Wroclaw Research Center EIT+ under the project "Biotechnologies and advanced medical technologies-BioMed" (POIG 01.01.02-02-003/0800) financed from the European Regional Development Fund (Operational Programme Innovative Economy, 1.1.2).

Open Access This article is distributed under the terms of the Creative Commons Attribution License which permits any use, distribution, and reproduction in any medium, provided the original author (s) and the source are credited.

\section{References}

1. Jemal A, Bray F, Center MM, Ferlay J, Ward E, Forman D (2011) Global cancer statistics. CA Cancer J Clin 61:69-90

2. Li F, Lai M (2009) Colorectal cancer, one entity or three. J Zhejiang Univ Sci B 10:219-29

3. Albuquerque C, Bakker ER, van Veelen W, Smits R (2011) Colorectal cancers choosing sides. Biochim Biophys Acta 1816:219-31

4. Papagiorgis PC, Zizi AE, Tseleni S, Oikonomakis IN, Sofras L, Elemenoglou IS, Patsouris E, Nikiteas NI (2011) Site impact on colorectal cancer biological behavior in terms of clinicopathological and molecular features. J BUON 16:84-92

5. Derwinger K, Gustawsson B (2011) Variations in demography and prognosis by colon cancer location. Anticancer Res 31:2347-50

6. Krzystek-Korpacka M, Matusiewicz M, Banas T (2006) Structure and function of midkine, a novel heparin-binding growth factor. Post Hig Med Dosw 60:591-601 
7. Muramatsu T (2010) Midkine, a heparin-binding cytokine with multiple roles in development, repair and diseases. Proc Jpn Acad Ser B Phys Biol Sci 86:410-25

8. Aridome K, Tsutsui J, Takao S, Kadomatsu K, Ozawa M, Aikou T, Muramatsu T (1995) Increased midkine gene expression in human gastrointestinal cancers. Jpn J Cancer Res 86:655-61

9. Miyashiro I, Kaname T, Nakayama T, Nakamori S, Yagyu S, Monden T, Kikkawa N, Nishisho I, Muramatsu T, Monden M, Akiyama T (1996) Expression of truncated midkine in human colorectal cancers. Cancer Lett 106:287-91

10. Aridome K, Takao S, Kaname T, Kadomatsu K, Natsugoe S, Kijima F, Aikou T, Muramatsu T (1998) Truncated midkine as a marker of diagnosis and detection of nodal metastases in gastrointestinal carcinomas. Br J Cancer 78:472-7

11. Ye C, Qi M, Fan Q-W, Ito K, Akiyama S, Kasai Y, Matsuyama M, Muramatsu T, Kadomatsu K (1999) Expression of midkine in the early stage of carcinogenesis in human colorectal cancer. Br J Cancer 79:179-84

12. Takuyama W, Mikami T, Fujiwara M, Matsui T, Okayasu I (2007) Midkine expression in colorectal tumors: correlation with Ki-67 labeling in sporadic but not ulcerative colitis-associated ones. Path Intern 57:260-7

13. Sobin LH, Wittekind C (2002) TNM classification of malignant tumors, 6th edn. Wiley, Hoboken

14. Bradford MM (1976) A rapid and sensitive method for the quantitation of microgram quantities of protein utilizing the principle of protein-dye binding. Anal Biochem 72:248-54

15. Song X-J, Muramatsu H, Aridome K, Aikou T, Koide N, Tsuji T, Muramatsu T (1997) The serum level of midkine, a heparinbinding growth factor, as a tumor marker. Biomed Res 18:375-381

16. Krzystek-Korpacka M, Matusiewicz M, Diakowska D, Grabowski K, Blachut K, Kustrzeba-Wojcicka I, Banas T (2007) Serum midkine depends on lymph node involvement and correlates with circulating VEGF-C in oesophageal squamous cell carcinoma. Biomarkers 12:403-413

17. Tanabe K, Matsumoto M, Ikematsu S, Nagase S, Hatakeyama A, Takano T, Niikura H, Ito K, Kadomatsu K, Hayashi S, Yaegashi N (2008) Midkine and its clinical significance in endometrial carcinoma. Cancer Sci 99:1125-30

18. Birkenkamp-Demtroder K, Olesen SH, Sørensen FB, Laurberg S, Laiho P, Aaltonen LA, Ørntoft TF (2005) Differential gene expression in colon cancer of the caecum versus the sigmoid and rectosigmoid. Gut 54:374-384

19. Minoo P, Zlobec I, Peterson M, Terracciano L, Lugli A (2010) Characterization of rectal, proximal and distal colon cancers based on clinicopathological, molecular and protein profiles. Int J Oncol 37:707-18

20. Kalady MF, Sanchez JA, Manilich E, Hammel J, Casey G, Church JM (2009) Divergent oncogenic changes influence survival differences between colon and rectal adenocarcinomas. Dis Colon Rectum 52:1039-45

21. Colombo C, Creighton CJ, Ghadimi MP, Bolshakov S, Warneke CL, Zhang Y, Lusby K, Zhu S, Lazar AJ, West RB, van de Rijn M, Lev D (2011) Increased midkine expression correlates with desmoid tumour recurrence: a potential biomarker and therapeutic target. J Pathol 225:574-82

22. Muramatsu T (2011) Midkine: a promising molecule for drug development to treat diseases of the central nervous system. Curr Pharm Des 17:410-23

23. Dai LC, Yao X, Wang X, Niu SQ, Zhou LF, Fu FF, Yang SX, Ping JL (2009) In vitro and in vivo suppression of hepatocellular carcinoma growth by midkine-antisense oligonucleotide-loaded nanoparticles. World J Gastroenterol 15:1966-72 